\title{
CONDUTÂNCIA Do DOSSEL, CONDUTÂNCIA AERODINÂMICA E FATOR DE DESACOPLAMENTO EM FLORESTA DE VOCHYSIA DIVERGENS POHL (VOCHYSIACEAE) NO PANTANAL BRASILEIRO
}

\author{
CAMILA ISABEL DE MENEZES FRAGA, LUCIANA SANCHES, OSVALDO BORGES PINTO \\ JUNIOR, LEONE FRANCISCO AMORIM CURADO, DENILTON CARLO GAIO
}
Universidade Federal de Mato Grosso, Programa de Pós-Graduação em Física Ambiental, Cuiabá, MT, Brasil
camilasanitarista@hotmail.com, 1sanches@ufmt.br,osvaldo.borges@gmail.com, leone@pgfma.ufmt.br, dcgaio@ufmt.br

Recebido Dezembro de 2013 - Aceito Outubro de 2014

\begin{abstract}
RESUMO
Neste trabalho avaliaram-se os mecanismos de controle da evapotranspiração por meio da condutância do dossel, condutância aerodinâmica e fator de desacoplamento em floresta de Vochysia divergens Pohl (Vochysiaceae) localizada no Mato Grosso no Pantanal Brasileiro. Durante o ano 2007 foram estimadas as variáveis: evapotranspiração, condutância do dossel, condutância aerodinâmica e pressão de vapor d'água. $\mathrm{O}$ fator de desacoplamento foi 0,58 e 0,51 durante as estações úmida e seca, respectivamente, indicando que o controle exercido pelo saldo de radiação sobre a evapotranspiração é predominante. $\mathrm{O}$ desacoplamento entre a cobertura vegetal e a atmosfera durante a estação úmida foi semelhante a estudos em florestas tropicais. Estes resultados acrescentam uma dimensão importante para a compreensão das características desta floresta inundável reconhecida internacionalmente.

Palavras-chave: áreas alagáveis; cambarazal; evapotranspiração.

ABSTRACT: CANOPY CONDUCTANCE, AERODYNAMIC CONDUCTANCE AND THE DECOUPLING COEFFICIENT IN THE VOCHYSIA DIVERGENS POHL (VOCHYSIACEAE) FOREST IN THE BRAZILIAN PANTANAL

In this study the control mechanisms of evapotranspiration through canopy conductance, aerodynamic conductance and decoupling coefficient for a Vochysia divergens Pohl (Vochysiaceae) forest located in the State of Mato Grosso in the Brazilian Pantanal are assessed. Over the course of the 2007 year, estimates of the following biophysical variables were made: evapotranspiration, canopy conductance, aerodynamic conductance and vapor pressure deficit. The decoupling coefficient was 0.58 and 0.51 for wet and dry season, respectively, indicating that the control offered by the net radiation on the evapotranspiration is predominant. The decoupling between the vegetation cover and atmosphere during the wet season was similar to studies in tropical forests. These results add an important dimension to our understanding on the characteristics of this internationally important wetland.
\end{abstract}

Keywords: cambarazal; evapotranspiration; wetlands.

\section{INTRODUÇÃ̃O}

As taxas de trocas de vapor d'água $\left(\mathrm{H}_{2} \mathrm{O}\right)$ entre a superfície vegetada e a atmosfera estão entre os principais componentes das trocas de energia nos processos que ocorrem na interface biosfera e atmosfera (Kumagai et al., 2004). Essa transferência do vapor de água do interior da estrutura celular das plantas para a atmosfera é regulada pela abertura dos estômatos presentes nas folhas, sendo otimizada no máximo de absorção de dióxido de carbono contra o mínimo de perdas de água (Wright et al., 1995).

A transpiração vegetal juntamente com a evaporação do solo, é parte dos mecanismos de evapotranspiração de superfícies vegetadas (Souza Filho, 2002). Variações na evapotranspiração são controladas pela demanda evaporativa atmosféricas, que determinam a taxa de evaporação potencial 
sob certas condições atmosféricas e pelo controle proveniente da superfície terrestre, que fisiológica e aerodinamicamente regula a evapotranspiração por meio de características como a atividade dos estômatos e rugosidade do dossel (Matsumoto et al., 2008).

A capacidade da superfície em transmitir água para a atmosfera é a condutância (Kelliher et al., 1995). Ao nível individual das folhas dos vegetais, o controle das trocas gasosas é quantificado pela condutância estomática $\left(\mathrm{C}_{\mathrm{e}}\right)$ (Takagi et al., 1998), que determina o limite da taxa de entrada de um fluxo de gás no poro estomático (Larcher, 2006).

Para o dossel, a capacidade de transmitir água para a atmosfera pode ser expressa pela condutância de superfície $\left(\mathrm{C}_{\mathrm{s}}\right)$, parâmetro determinado na prática por medidas de evapotranspiração (ou de transpiração) e de outros parâmetros meteorológicos atuantes sobre a vegetação (Takagi et al., 1998).

As taxas de momentum, fluxo de calor latente e água trocadas entre o dossel vegetal e a camada superficial atmosférica, assim como folha/superfície do solo e o ar em torno, são descritas utilizando as condutâncias aerodinâmica $\left(C_{a}\right)$ e superficial $\left(\mathrm{C}_{\mathrm{s}}\right)$ que representam, respectivamente, a capacidade de conduzir fluxo de ar acima da superfície vegetativa e fluxo do vapor de água de uma superfície evaporante (estômato vegetal e solo) (Lecina et al., 2003)

A transferência de vapor no interior do dossel é dependente da velocidade do vento, ou indiretamente da $C_{a}$, que segundo Grelle et al. (1999) é função de parâmetros de turbulência enquanto a $\mathrm{C}_{\mathrm{e}}$ é, principalmente, controlada pelas variáveis da evaporação como a radiação global e o déficit de pressão de vapor. $\mathrm{AC}_{\mathrm{a}}$ representa as condições físicas do dossel e ao longo deste, e o controle da transpiração do dossel como um todo, que pode ser influenciada pelas condições climáticas. Por exemplo, os maiores valores na velocidade do vento são favoráveis ao aumento da evapotranspiração, pois o processo de transferência de vapor d'água é facilitado em função do aumento da $\mathrm{C}_{\mathrm{a}}$ (Souza Filho, 2002).

De acordo com Stewart (1988), para a estimativa da transpiração a partir de observações meteorológicas, a equação de Penman-Monteith é frequentemente utilizada e para tanto, o complexo arranjo da $\mathrm{C}_{\mathrm{e}}$ de folhas individuais de uma floresta e a associada $C_{a}$ são substituídos por um sistema equivalente constituído por uma única folha hipotética, denominada "grande folha" com uma única $\mathrm{C}_{\mathrm{e}}$ e $\mathrm{C}_{\mathrm{a}}$. A partir desta equação, Mcnaughton e Jarvis (1983) desenvolveram o conceito do fator de desacoplamento $(0 \leq \Omega \leq 1)$, que é um indicativo da interação biosfera-atmosfera nos processos de evapotranspiração vegetal. Quando igual ou próximo a 0 , o controle biótico sobre a evapotranspiração é grande, e quando próximo a 1 , as condições externas são determinantes no processo.
O grau de acoplamento entre a folha ou o dossel e atmosfera determina se a taxa de transpiração é principalmente controlada pelo déficit de pressão de vapor e a condutância do estômato (forte acoplamento) ou se pelo saldo de radiação (fraco acoplamento) (Daudet et al., 1999).

Neste trabalho objetivou-se avaliar os mecanismos de controle da evapotranspiração por meio da condutância do dossel, condutância aerodinâmica e fator de desacoplamento em floresta de Vochysia divergens Pohl (Vochysiaceae) localizada no Mato Grosso no Pantanal.

\section{MATERIAL E MÉTODOS}

\subsection{Descrição e localização da área de estudo}

Este estudo foi conduzido na Reserva Particular do Patrimônio Nacional (RPPN) SESC Pantanal município de Barão de Melgaço, Mato Grosso, Brasil, em área de Vockysia Divergens Pohl (localmente conhecido como cambarazal (DamascenoJúnior et al., 2005), sazonalmente inundada inserida na planície fisiográfica do Pantanal, sob altitude média de $120 \mathrm{~m}$, a $165 \mathrm{~km}$ de Cuiabá. Nesta área foi instalada uma torre micrometerológica

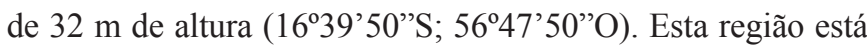
sob domínio climático Aw, segundo a classificação de Köppen, com duas estações bem definidas, uma seca de abril a outubro, e outra úmida, de novembro a março.

\subsection{Instrumentação}

Os dados das variáveis meteorológicas medidos por equipamentos instalados na torre foram utilizados nos cálculos da condutância aerodinâmica, condutância de superfície e do fator de desacoplamento.

Sensores meteorológicos foram instalados em uma torre de $32 \mathrm{~m}$ de altura para medições que ocorreram de fevereiro de 2007 a janeiro de 2008. As medidas de saldo de radiação e de radiação solar incidente foram obtidas por meio de um sensor Net Radiometer Kipp e Zonen Delft Inc. Holland ${ }^{\mathbb{B}}$ e de um piranômetro LI-200 Campbell Sci Inc. USA ${ }^{\circledR}$, respectivamente, instalados a $33 \mathrm{~m}$ com o auxílio de um braço metálico com o objetivo de não ser influenciado pela própria estrutura da torre.

A temperatura e a umidade relativa do ar foram obtidas dos registros de dois termohigrômetros HMP 45 C Vaisala Inc. Helsinki Finland ${ }^{\circledR}$ instalados nas alturas de $33,7 \mathrm{~m}$ e $37,7 \mathrm{~m}$. A velocidade do vento foi obtida por anemômetro de chonchas A-014 Wind Speed Sensor Met One Instruments, Inc., USA ${ }^{\circledR}$, instalado a 33,7 de altura do solo.

Adjacente a torre micrometeorológica foi instalada uma placa de fluxo de calor no solo HFT-3.1 REBS Inc. Seattle Washington ${ }^{\circledR}$ a $2 \mathrm{~cm}$ de profundidade. 
Os dados de precipitação foram medidos no posto de monitoramento climatológico da RPPN SESC Pantanal de Porto Cercado distante $15 \mathrm{~km}$ da torre micrometeorológica.

Os sensores micrometeorológicos e de medição de fluxo de calor no solo e da temperatura do solo estavam conectados a um sistema de controle e registro acoplado a um multiplexador modelo AM 16 Cambpell Scientific Inc. Logan UT USA ${ }^{\circledR}$, alimentado por um conjunto de baterias mantidas carregadas por meio de painéis solares modelo SP 65 Cambpell Scientific Inc. Logan UT USA ${ }^{\circledR}$. As médias dos dados coletados foram armazenadas a cada 15 minutos em um datalogger MM900 ELE International Bedfordshire $\mathrm{UK}^{\circledR} \mathrm{e}$ transferidas para o disco rígido de um computador a cada quinze dias.

\subsection{Estimativa do fluxo de calor latente e sensível}

O fluxo de calor latente e sensível foi estimado a partir do balanço de energia à superfície terrestre, descrito pela Equação 1 .

$$
\mathrm{Rn}=\mathrm{G}+\mathrm{H}+\mathrm{LE}
$$

Em que Rn é o saldo de radiação ( $\mathrm{W} \mathrm{m}^{-2}$ ), $\mathrm{G}$ é o fluxo de calor no solo $\left(\mathrm{W} \mathrm{m}^{-2}\right)$, H é o fluxo de calor sensível $\left(\mathrm{W} \mathrm{m}^{-2}\right)$ e LE é o fluxo de calor latente $\left(\mathrm{W} \mathrm{m}^{-2}\right)$.

$\mathrm{O}$ LE e o $\mathrm{H}$ foram estimados a partir das Equações $2 \mathrm{e}$ 3 , respectivamente.

$$
\begin{aligned}
& L E=\frac{R n-G}{1+B} \\
& H=\frac{B}{1+B}(R n-G)
\end{aligned}
$$

Para caracterizar a partição da energia disponível a partir dos registros dos gradientes de temperatura e de pressão de vapor acima da superfície evaporante foi utilizada a razão de Bowen (B) e assumidas as suposições teóricas que se basearam em Todd et al. (2000). Para que estas premissas fossem cumpridas, considerou-se que a borda de ataque ao efeito do vento (fetch) fossem suficientes, o que segundo Monteith e Unsworth (1990) deve ser da ordem de $100 \mathrm{~m}$ na horizontal para cada $1 \mathrm{~m}$ de medição na vertical conforme Equação 4.

$$
\mathrm{B}=\frac{\mathrm{H}}{\mathrm{LE}}=\gamma \frac{\Delta \mathrm{T}}{\Delta \mathrm{e}}
$$

Em que, $\gamma$ é o coeficiente psicrométrico $\left(\mathrm{kPa}^{\circ} \mathrm{C}^{-1}\right), \Delta \mathrm{T}$ e $\Delta \mathrm{e}$, respectivamente, as diferenças de temperatura do ar vertical $\left({ }^{\circ} \mathrm{C}\right)$ e de pressão de vapor d'água $(\mathrm{kPa})$ em dois níveis.

O coeficiente psicrométrico foi determinado a partir da Equação 5.

$$
\gamma=0,0016286 \frac{\mathrm{P}}{\lambda}
$$

Em que $\mathrm{P}$ é a pressão atmosférica $(\mathrm{kPa})$ e $\lambda$ é o calor latente de evaporação $\left(\mathrm{MJ} \mathrm{kg}^{-1}\right)$.

A pressão atmosférica foi determinada de acordo com Burman et al. (1987), Equação 6.

$$
\mathrm{P}=\mathrm{P}_{0}\left[\frac{\mathrm{T}_{\mathrm{k} 0}-\mathrm{r}\left(\mathrm{A}-\mathrm{z}_{\mathrm{p}}\right)}{\mathrm{T}_{\mathrm{k} 0}}\right]^{\frac{\mathrm{g}}{\mathrm{rR}}}
$$

Em que, $\mathrm{P}$ é a pressão atmosférica $(\mathrm{kPa}), \mathrm{P}_{0}$ é a pressão atmosférica ao nível do $\operatorname{mar}(101,3 \mathrm{kPa})$, r é a razão adiabática para o ar saturado $\left(0,0065 \mathrm{~K} \mathrm{~m}^{-1}\right)$, A é a altitude $(120 \mathrm{~m}), \mathrm{z}_{\mathrm{p}}$ é a altura de medição da pressão $\mathrm{P}(\mathrm{m})$, g é a aceleração da gravidade $\left(9,8 \mathrm{~m} \mathrm{~s}^{-2}\right)$, $\mathrm{R}$ é a constante específica do gás para o ar seco $\left(287 \mathrm{~J} \mathrm{~kg}^{-1} \mathrm{~K}^{-1}\right)$ e $\mathrm{T}_{\mathrm{k} 0}$ é a temperatura absoluta medida no topo da torre $(\mathrm{K})$.

Para a determinação do calor latente de evaporação, considerou-se a Equação 7.

$$
\lambda=2,501-\left(2,361 \times 10^{-3}\right) \times \mathrm{T}
$$

Em que, $\mathrm{T}$ é a temperatura do ar $\left({ }^{\circ} \mathrm{C}\right)$.

A diferença de pressão de vapor entre dois níveis $(\Delta \mathrm{e})$ foi determinada de acordo com Shuttleworth (2012), Equação 8.

$$
\Delta \mathrm{e}=\Delta \mathrm{e}_{\mathrm{su}}-\gamma\left(\Delta \mathrm{T}_{\mathrm{s}}\right)+\gamma\left(\Delta \mathrm{T}_{\mathrm{u}}\right)
$$

Em que, $\Delta \mathrm{e}_{\mathrm{su}}, \Delta \mathrm{T}_{\mathrm{s}}$ e $\Delta \mathrm{T}_{\mathrm{u}}$ são as diferença de pressão de saturação na temperatura do bulbo úmido $(\mathrm{kPa})$, de temperatura do bulbo seco $\left({ }^{\circ} \mathrm{C}\right)$ e de temperatura do bulbo úmido $\left({ }^{\circ} \mathrm{C}\right)$ entre dois níveis, respectivamente.

A pressão de saturação de vapor d'água para o bulbo úmido $\left(\mathrm{e}_{\mathrm{su}}\right)$ foi determinada conforme a Equação 9.

$$
\mathrm{e}_{\mathrm{su}}=0,6108 \times \exp \left[\frac{17,27 \times \mathrm{T}_{\mathrm{u}}}{\mathrm{T}_{\mathrm{u}}+237,3}\right]
$$

Em que, $\mathrm{T}_{\mathrm{u}}$ é a temperatura do bulbo úmido $\left({ }^{\circ} \mathrm{C}\right)$, que foi determinada pela Equação 10.

$$
\mathrm{T}_{\mathrm{u}}=\frac{\mathrm{e}-\mathrm{e}_{\mathrm{s}}+(\gamma * \mathrm{UR})}{\gamma}
$$

Em que, UR é umidade relativa do ar (\%). Os dados utilizados nas Eqs 8-10 foram medidos por termohigrômetro descrito no item 2.2, os termohigrômetros são equipamentos amplamente utilizados em estudos de balanço de energia e de massa em ecossistemas tropicais (Sanches et al. 2011; Vourlitis et al. 2008; Priante-Filho et al., 2014).

Para os valores de razão de Bowen seguiram as recomendações de Ortega-Farias et al. (1996) e Unland et al. (1996). Além disso, as consistências dos fluxos de LE e H obtidos pela razão de Bowen foram avaliadas conforme Perez et al. (1999).

Para o dia médio de evapotranspiração real $\left(E T_{r}\right)$, déficit de pressão de vapor d'água (DPV), condutância de superfície 
$\left(\mathrm{C}_{\mathrm{s}}\right)$, condutância aerodinâmica $\left(\mathrm{C}_{\mathrm{a}}\right)$ e fator de desacoplamento $(\Omega)$ foram consideradas as estimativas entre as $7 \mathrm{e}$ as 18 horas.

\subsection{Estimativa dos mecanismos de controle da evapotranspiração}

Para a compreensão dos mecanismos de controle da evapotranspiração em $V$. divergens foi utilizado o conceito de condutância, que distingue os fatores microclimáticos ou abióticos dos efeitos bióticos presentes na vegetação.

A condutância aerodinâmica $\left(\mathrm{C}_{\mathrm{a}}\right)$, o Rn e o déficit de pressão de vapor d'água na atmosfera (DPV) representam os fatores microclimáticos. Os fatores bióticos são representados pela condutância de superfície $\left(\mathrm{C}_{\mathrm{s}}\right)$ e ao controle exercido pelos estômatos sobre a transmissão da água para a atmosfera.

Para a determinação da condutância aerodinâmica $\left(\mathrm{C}_{\mathrm{a}}\right)$ considerou-se a Equação 11:

$$
\mathrm{C}_{\mathrm{a}}=\frac{0,4^{2} \mathrm{u}(\mathrm{z})}{\left[\ln \left(\frac{\mathrm{z}-\mathrm{d}}{\mathrm{z}_{0}}+\Phi_{\mathrm{m}}\right)\right]^{2}}
$$

Em que, $\mathrm{C}_{\mathrm{a}}$ é a condutância aerodinâmica $\left(\mathrm{m} \mathrm{s}^{-1}\right)$, u é a velocidade do vento $\left(\mathrm{m} \mathrm{s}^{-1}\right)$ na altura de medição $\mathrm{z}(\mathrm{m}), \mathrm{d} o$ deslocamento do plano zero $(\mathrm{m}), \mathrm{z}_{0}$ a rugosidade da superfície (m). $\Phi_{\mathrm{m}}$ correspondem ao fator de correção do perfil do vento.

Os valores de $\mathrm{z}_{0}$ e d considerados nesta área de floresta de $V$. divergens foram $0,10 \mathrm{~h}$ e $0,70 \mathrm{~h}$, respectivamente, em que $\mathrm{h}$ corresponde a $29 \mathrm{~m}$, a altura média do dossel e foram adaptados a partir de Shuttleworth (1988) e Campbell e Norman (1998).

As condições de estabilidade ou instabilidade atmosféricas são mais facilmente detectadas por meio do número de Richardson $\left(\mathrm{R}_{\mathrm{i}}\right)$, que compara os efeitos das forças térmicas, responsáveis pela convecção livre, com aqueles das forças mecânicas, causadoras de convecção forçada (Pereira, 2000). Portanto, para situações de não neutralidade atmosférica, determinou-se $\mathrm{R}_{\mathrm{i}}$ conforme a Equação 12.

$$
\mathrm{R}_{\mathrm{i}}=\frac{\mathrm{g}\left(\frac{\Delta \mathrm{T}}{\Delta \mathrm{z}}\right)}{\mathrm{T}\left(\frac{\Delta \mathrm{u}}{\Delta \mathrm{z}}\right)^{2}}
$$

Em que, $\mathrm{R}_{\mathrm{i}}$ é o número de Richardson (adimensional), g, a aceleração da gravidade $\left(9,8 \mathrm{~m} \mathrm{~s}^{-2}\right), \Delta \mathrm{T}$, a diferença de temperatura do ar $\left({ }^{\circ} \mathrm{C}\right), \Delta \mathrm{u}$, a diferença de velocidade do vento $\left(\mathrm{m} \mathrm{s}^{-1}\right), \mathrm{T}$, a temperatura potencial média $(\mathrm{K}) \mathrm{e} \Delta \mathrm{z}$, a diferença entre as alturas de medição.

De acordo com Pereira (2000) o sinal de $\Delta \mathrm{T} / \Delta \mathrm{z}$ controla o sinal de $\mathrm{R}_{\mathrm{i}}$, que pode ser maior, menor ou igual a zero. Em condições de neutralidade atmosférica, $\mathrm{R}_{\mathrm{i}}$ é igual a zero, valores positivos indicam estabilidade e valores negativos, atmosfera instável.

Para a correção do perfil de vento em condições de não neutralidade utilizou-se a função $\Phi_{\mathrm{m}}$ segundo a proposta adotada por Righi (2004):

Em condições estáveis $\left(1>R_{i}>0,01\right)$, foi utilizada a Equação 13.

$$
\Phi_{\mathrm{m}}=\left(1-5,2 \mathrm{R}_{\mathrm{i}}\right)^{2}
$$

Em condições neutras $\left(-0,01 \geq R_{i} \leq 0,01\right)$, adotou-se a Equação 14.

$$
\Phi_{\mathrm{m}}=1
$$

Em condições instáveis $\left(-0,01>\mathrm{R}_{\mathrm{i}}>-1\right)$, foi utilizada a Equação 15.

$$
\Phi_{\mathrm{m}}=\left(1-16 \mathrm{R}_{\mathrm{i}}\right)^{0,75}
$$

Segundo Kelliher et al. (1995), quando o índice de área foliar (IAF) é superior a $3 \mathrm{~m}^{2} \mathrm{~m}^{-2}$ a evaporação do solo é tão pequena que a determinação micrometeorológica da condutância de superfície $\left(\mathrm{C}_{\mathrm{s}}\right)$ permite uma boa aproximação do parâmetro fisiológico condutância estomática $\left(\mathrm{C}_{\mathrm{e}}\right)$. Dessa maneira, a partir dos resultados prévios referentes as condições da superfície vegetada, a $\mathrm{C}_{\mathrm{s}}$ foi calculada utilizando a equação invertida de Penman-Monteith, Equação 16.

$$
\mathrm{C}_{\mathrm{s}}=\left(\mathrm{r}_{\mathrm{s}}\right)^{-1}=\left[\frac{\rho_{\mathrm{a}} \mathrm{c}_{\mathrm{p}} \mathrm{DPV}}{\gamma \mathrm{LE}}-\frac{1}{\mathrm{C}_{\mathrm{a}}}\left(1-\frac{\mathrm{sH}}{\gamma \mathrm{LE}}\right)^{-1}\right]
$$

Em que, $\mathrm{C}_{\mathrm{s}}$ é a condutância de superfície $\left(\mathrm{m} \mathrm{s}^{-1}\right), \mathrm{r}_{\mathrm{s}}$ é a resistência da superfície $\left(\mathrm{m} \mathrm{s}^{-1}\right), \rho_{\mathrm{a}}$ é a densidade do ar $(\mathrm{Kg}$ $\left.\mathrm{m}^{-3}\right), \mathrm{c}_{\mathrm{p}}$ é o calor específico do ar úmido $\left(1013 \mathrm{~J} \mathrm{Kg}^{-1}{ }^{\circ} \mathrm{C}^{-1}\right)$, DPV é o déficit de pressão de vapor $(\mathrm{kPa}), \gamma$ é a constante psicrométrica $\left(\mathrm{kPa}^{\circ} \mathrm{C}^{-1}\right)$, LE é o fluxo de calor latente $\left(\mathrm{W} \mathrm{m}^{-2}\right)$, $\mathrm{C}_{\mathrm{a}}$ é a condutância aerodinâmica $\left(\mathrm{m} \mathrm{s}^{-1}\right), \mathrm{H}$ é o fluxo de calor sensível $\left(\mathrm{W} \mathrm{m}^{-2}\right)$ e s é a declividade da curva de pressão de saturação de vapor d'água $\left(\mathrm{kPa}^{\circ} \mathrm{C}^{-1}\right)$.

O DPV foi determinado a partir da Equação 17.

$$
\mathrm{DPV}=\mathrm{e}_{\mathrm{s}}-\mathrm{e}_{\mathrm{a}}
$$

Em que, e é a pressão de saturação de vapor d'água $(\mathrm{kPa}) \mathrm{e}, \mathrm{e}_{\mathrm{a}}$ a pressão atual de vapor d'água $(\mathrm{kPa})$.

A declividade da curva de pressão saturação (s) foi estimada a partir da Equação 18.

$$
\mathrm{s}=\frac{4098 \mathrm{e}_{\mathrm{s}}}{(\mathrm{T}+237,3)^{2}}
$$

Em que, $e_{\mathrm{s}}$ é a pressão de saturação de vapor d'água (kPa) e Té a temperatura do ar $\left({ }^{\circ} \mathrm{C}\right)$. 
A transpiração vegetal pode depender tanto das características do dossel, quanto das condições da atmosfera local. A interação entre o dossel e atmosfera foi calculada de acordo com a teoria do fator de desacoplamento $(\Omega)$ de McNaughton e Jarvis (1983), Equação 19.

$$
\Omega=\frac{1}{1+[\gamma /(\mathrm{s}+\gamma)]\left(\mathrm{C}_{\mathrm{a}} / \mathrm{C}_{\mathrm{s}}\right)}
$$

O $\Omega$ varia conforme o intervalo $0<\Omega<1$, sendo que valores próximos ou iguais a 0 , indicam que a transpiração vegetal depende do controle dos estômatos e, quando próximos a 1, que a transpiração está sensível ao saldo de radiação solar.

\section{RESULTADOS E DISCUSSÕES}

\subsection{Mecanismos de controle da evapotranspiração}

A Figura 1 apresenta o dia médio da $\mathrm{ET}_{\mathrm{r}}$, Rn e DPV entre as 7 e 18 horas durante as estações seca e úmida. A elevada disponibilidade energética associada à umidade do solo suficiente e acessível propiciaram taxas significativas de $\mathrm{ET}_{\mathrm{r}}$, tanto nos períodos secos, quanto nos úmidos. A variabilidade do dia médio de $\mathrm{ET}_{\mathrm{r}}$ de cada estação foi similar a verificada para o Rn, com pico máximo as 11 horas no valor de $0,75 \mathrm{~mm}$ $\mathrm{h}^{-1}$ na estação úmida.

Em relação a $\mathrm{ET}_{\mathrm{r}}$ não foi verificada correlação significativa quanto ao DPV ( $\mathrm{n}=5288$; +CI 95\%). Na escala sazonal, observou-se aumento do DPV e diminuição da $\mathrm{ET}_{\mathrm{r}}$ na estação seca, este comportamento é indicativo de que o principal componente atuante sobre a $\mathrm{ET}^{\mathrm{r}}$ é o saldo de radiação, e ainda a possibilidade de que o mesmo possa ter sofrido alguma influência decorrente do método adotado para a estimativa de $\mathrm{ET}_{\mathrm{r}}$.

BUCCI et al. (2008) reportaram que para diferentes estandes de cerrado, não houve relação significativa entre a evaporação medida e o DPV, que apresentou comparativamente valores maiores na estação seca do que na úmida.

As máximas e mínimas médias estacionais de DPV foram $3,1 \mathrm{kPa}$ e $0,2 \mathrm{kPa}$, respectivamente, e ambas na estação seca. Estes valores estão de acordo com a variação de 0,2 a 2,3 kPa apresentada por BUCCI et al. (2008) em condições de Cerrado.

O DPV estacional em $V$. divergens atingiu valores máximos a partir das 12 horas, em todos os períodos observados, e foram negativos em relação as variações diurnas da $\mathrm{C}_{\mathrm{s}}$. Souza Filho (2002) apresentou DPV médio bem definido e inverso ao comportamento da $\mathrm{C}_{\mathrm{s}}$ para floresta Amazônica em Caxiuanã nos períodos chuvoso e menos chuvoso, com valores máximos aproximados de $1 \mathrm{kPa}$ e $1,6 \mathrm{kPa}$, respectivamente.

Foi verificado aumento da $\mathrm{C}_{\mathrm{s}}$ nas primeiras horas da manhã, com máximo de $0,024 \mathrm{~m} \mathrm{~s}^{-1}$ às 10 horas na estação seca e $0,026 \mathrm{~m} \mathrm{~s}^{-1}$ as 9 horas na estação úmida, com decréscimo de $\mathrm{C}_{\mathrm{s}}$ quase linear durante a tarde para ambos os períodos observados em $V$. divergens. Conforme Alves e Pereira (2000), o aumento acentuado da $\mathrm{C}_{\mathrm{s}}$ nas primeiras horas da manhã está de acordo com a indução da abertura dos estômatos provocada pela luz solar, e a diminuição no período da tarde se deve a
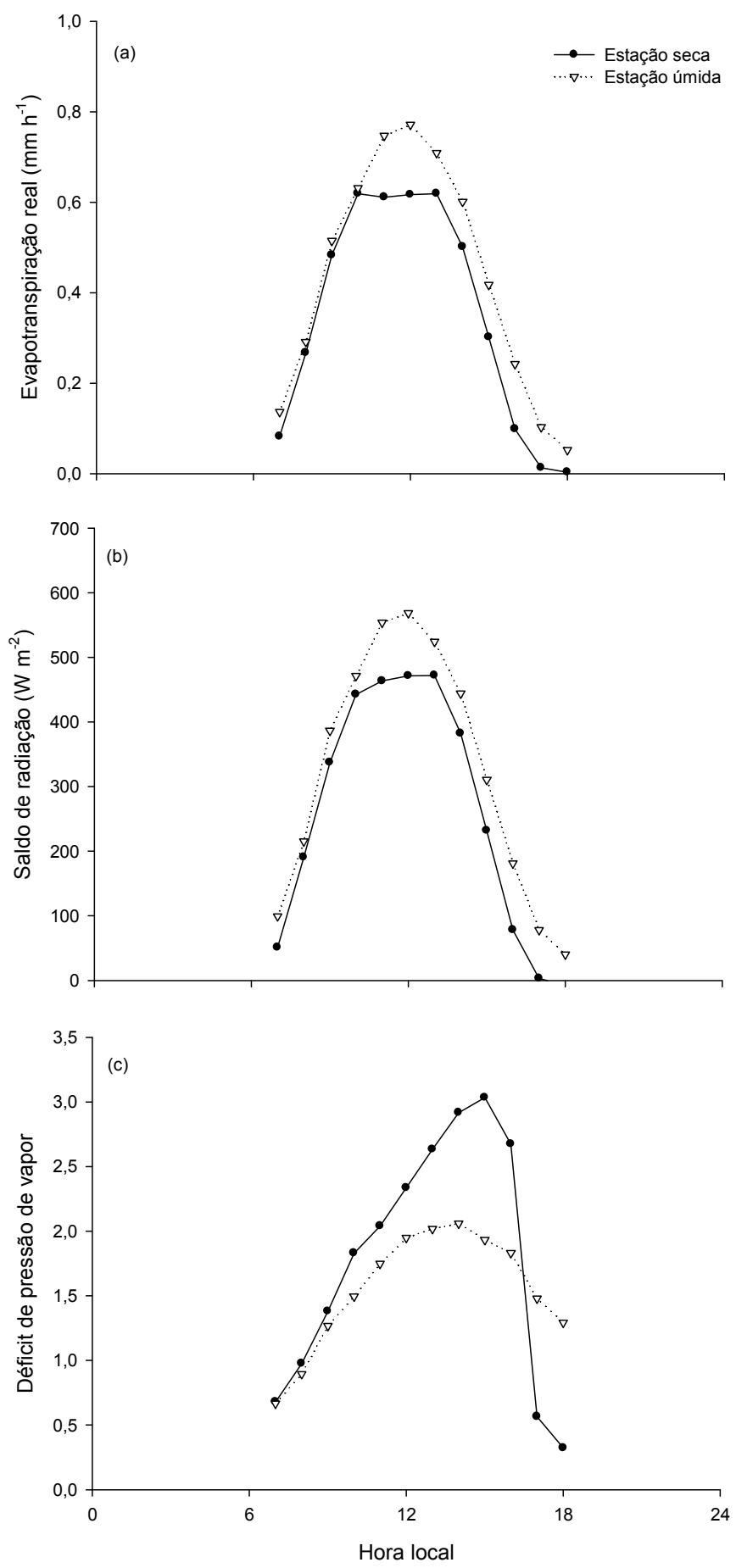

Figura 1: Dia médio estacional da (a) evapotranspiração real $\left(E_{r}\right)$, (b) saldo de radiação $(\mathrm{Rn})$ e (c) déficit de pressão de vapor (DPV). 
outras variáveis do microclima que influenciam a $\mathrm{C}_{\mathrm{s}}$, como a temperatura do ar e o DPV. Os picos horários máximos e valores de $\mathrm{C}_{\mathrm{s}}$ estimados em $V$. divergens estão de acordo com os apresentados por Wright et al. (1995), Granier et al. (1996), Souza Filho (2002) em estudos desenvolvidos em florestas tropicais e por San José et al. (2001) em uma área de floresta alagável na Venezuela.

Kumagai et al. (2004), em estudo desenvolvido em uma floresta tropical na Malásia, relataram que embora no período seco sejam notáveis os maiores valores de DPV, a magnitude da redução da $\mathrm{C}_{\mathrm{s}}$, com o aumento do DPV, foi maior no período úmido, para todos os valores de radiação solar considerados no estudo. Em $V$. divergens foi verificado comportamento semelhante para dia médio estacional do DPV e da $\mathrm{C}_{\mathrm{s}}$.

$\mathrm{Na}$ floresta de $V$. divergens, a maior sensibilidade da $\mathrm{C}_{\mathrm{s}}$ ocorreu no período vespertino, quando o DPV foi superior $2 \mathrm{kPa}$, tanto para estação seca, quanto para a úmida. Declínio semelhante na $\mathrm{C}_{\mathrm{s}}$ foi verificado por Wullschleger et al. (2000) no limiar de $2 \mathrm{kPa}$ para o DPV em área floresta de carvalho no Tennessee (EUA) no período da tarde.

Granier et al. (1996) em estudo sobre a transpiração de uma floresta tropical natural verificou a baixa dependência da condutância do dossel a radiação global, atribuindo este comportamento ao baixo limiar de saturação da luz, e que portanto, para o referido estudo, não foi fator limitante durante a maior parte do dia, enquanto que o déficit da pressão de vapor explicou quase que totalmente o comportamento da variação diária da condutância do dossel.

Em todo o período de observação, as estimativas de $\mathrm{C}_{\mathrm{a}}$ não ultrapassaram $0,05 \mathrm{~m} \mathrm{~s}^{-1}$ nos valores de dia médio estacional, e, considerando o intervalo entre as $7 \mathrm{~h}$ e as $18 \mathrm{~h}$, a $\mathrm{C}_{\mathrm{a}}$ média foi de $0,042 \mathrm{~m} \mathrm{~s}^{-1}$ e $0,038 \mathrm{~m} \mathrm{~s}^{-1}$ para a estação úmida e a seca, respectivamente, com variabilidade semelhante a verificada para o dia médio estacional da velocidade horizontal do vento (Figura 2).

Os dados mensais obtidos em $V$. divergens (Tabela 1) indicam uma diminuição da $\mathrm{ET}_{\mathrm{r}}$ de abril a setembro (estação seca), mesmo período em que ocorreu diminuição do saldo de radiação.

Entre março e outubro a $\mathrm{ET}_{\mathrm{r}}$ superou a precipitação na área em estudo. Comportamento semelhante foi observado em área de floresta tropical em Rondônia e em Mato Grosso por COSTA et al. (2007), de abril a setembro e entre maio e novembro, respectivamente.

A diferença entre os registros mensais de precipitação e as estimativas de $\mathrm{ET}_{\mathrm{r}}$ em $V$. divergens foi aproximadamente $13 \mathrm{~mm}$. Soriano e Galdino (2002) verificaram na sub-região da Nhecolândia, Pantanal de Mato Grosso do Sul, que a precipitação não foi suficiente para suprir a evapotranspiração potencial $(1477,2 \mathrm{~mm})$ solicitada pelo regime térmico em 2000 , resultando em déficit hídrico de $339,4 \mathrm{~mm}$, apesar da ocorrência de excedente hídrico anual de 295,8 $\mathrm{mm}$.

Em setembro, mês da estação seca, foram observados valores médios de 2,8 $\pm 1,1 \mathrm{kPa}$ para o DPV, os maiores verificados em $V$. divergens (Figura 3 ). $\mathrm{A}_{\mathrm{s}}$ entre agosto $\mathrm{e}$ setembro, apresentou menores médias mensais.
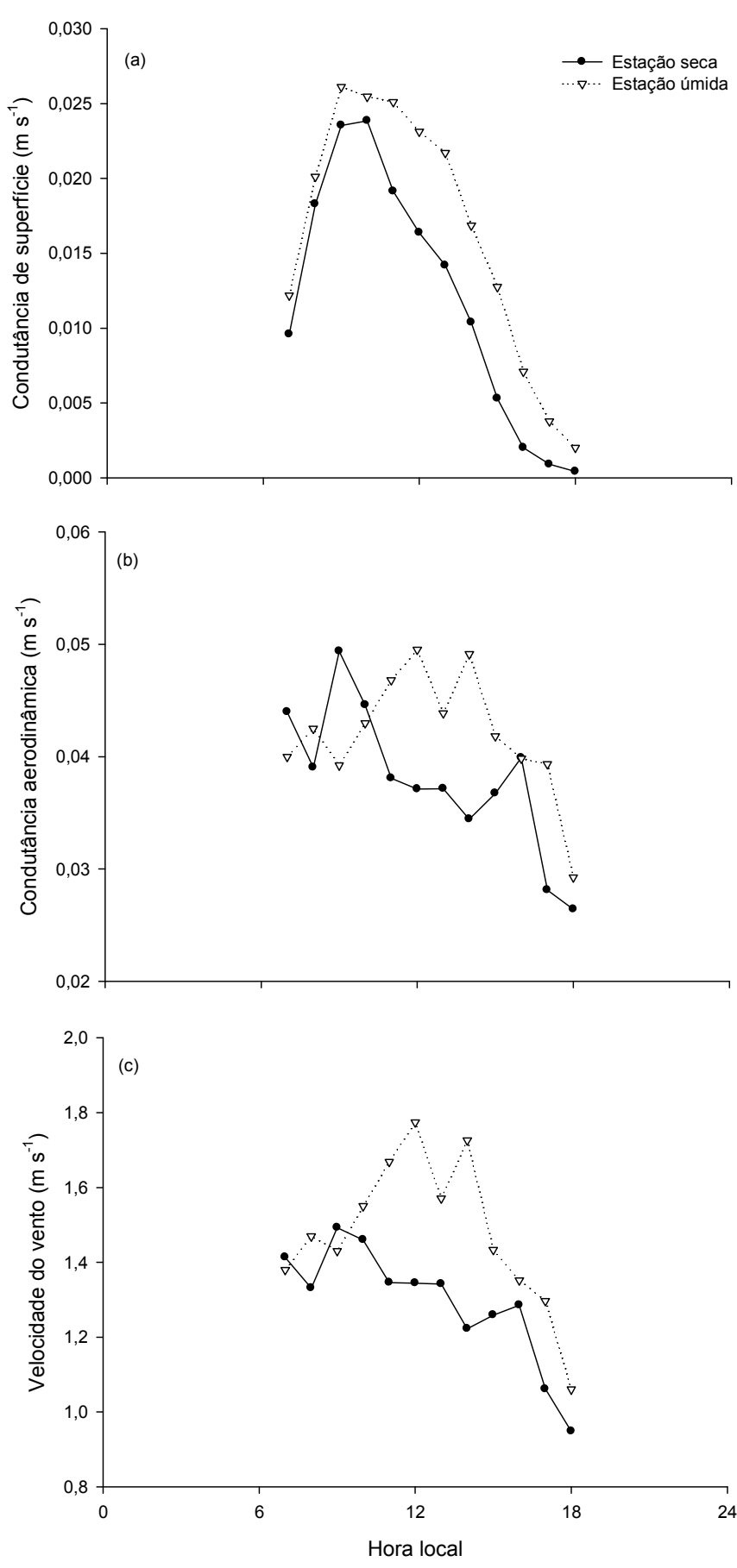

Figura 2: Variabilidade do dia médio estacional da (a) condutância de superfície $\left(\mathrm{C}_{\mathrm{s}}\right)$, (b) condutância aerodinâmica $\left(\mathrm{C}_{\mathrm{a}}\right)$ e $(\mathrm{c})$ velocidade do vento $(\mathrm{u})$ 
Tabela 1: Média diária da precipitação pluvial (Prec.) e evapotranspiração real (ETr).

\begin{tabular}{|c|c|c|c|}
\hline Estação & Mês & $\begin{array}{c}\text { Prec. } \\
\left(\mathrm{mm} \mathrm{d}^{-1}\right)\end{array}$ & $\begin{array}{c}\mathrm{ET}_{\mathrm{r}} \\
\left(\mathrm{mm} \mathrm{d}^{-1}\right)\end{array}$ \\
\hline Úmida & Fevereiro & 6,5 & 4,7 \\
\hline Úmida & Março & 2,2 & 5,2 \\
\hline Seca & Abril & 4,2 & 4,7 \\
\hline Seca & Maio & 1,3 & 3,2 \\
\hline Seca & Junho & 0,0 & 2,7 \\
\hline Seca & Julho & 1,3 & 2,7 \\
\hline Seca & Agosto & 0,0 & 3,4 \\
\hline Seca & Setembro & 0,0 & 2,7 \\
\hline Úmida & Outubro & 0,6 & 3,7 \\
\hline Úmida & Novembro & 10,6 & 4,3 \\
\hline Úmida & Dezembro & 12,7 & 5,2 \\
\hline Úmida & Janeiro & 7,9 & 4,4 \\
\hline & & & \\
\hline
\end{tabular}

\subsection{Fator de desacoplamento e parâmetros micrometeorológicos}

O coeficiente de desacoplamento $(\Omega)$ proposto por McNaughton e Jarvis (1983) e Jarvis e McNaughton (1986) foi estimado de maneira a distinguir a sensibilidade da evapotranspiração real $\left(\mathrm{ET}_{\mathrm{r}}\right)$ as variáveis físicas e a fatores fisiológicos em $V$. divergens. $\mathrm{O} \Omega$ varia entre 0 e 1 , sendo que, quanto mais próximo de 0 , maior é o acoplamento entre a superfície e a atmosfera, e mais efetivo é o controle estomático no processo de evapotranspiração. Valores próximos a 1 indicam que a evapotranspiração torna-se cada vez mais dependente do saldo de radiação recebido e menos dependente do controle estomático e do déficit de pressão de vapor (DPV).

$\mathrm{O} \Omega$ médio estimado em $V$. divergens durante a estação úmida foi 0,58 e para a seca, 0,51 . Ao longo do dia médio estacional as semelhanças entre os valores e picos se mantiveram para período úmido e para o seco, respectivamente com valores máximos de 0,70 e 0,66 durante a manhã e declínio após as 14 horas (Figura 4).

Souza Filho (2002) apresentou desacoplamento da floresta Amazônica localizada em Caxiuanã em relação a atmosfera, com valores horários médios de $\Omega$ maiores para o período chuvoso pela manhã e declínio durante a tarde.

$\mathrm{O} \Omega$ observado é indicativo da maior importância da energia disponível na condução da evapotranspiração durante a estação úmida, considerando relativamente menor a importância do controle estomático (Tabela 2). Comportamento semelhante foi observado em florestas tropicais associado ao índice de área foliar elevado (Pinto-Júnior et al., 2011; Souza Filho, 2002; Cienciala et al., 2000; Wullschleger et al., 1998; Meinzer et al., 1997). Em área de $V$. divergens, mesma área em estudo característica foi verificada, com IAF médio anual de 4,3 $\mathrm{m}^{2} \mathrm{~m}^{-2}$ (Sanches et al., 2011). De acordo com Meinzer et al.
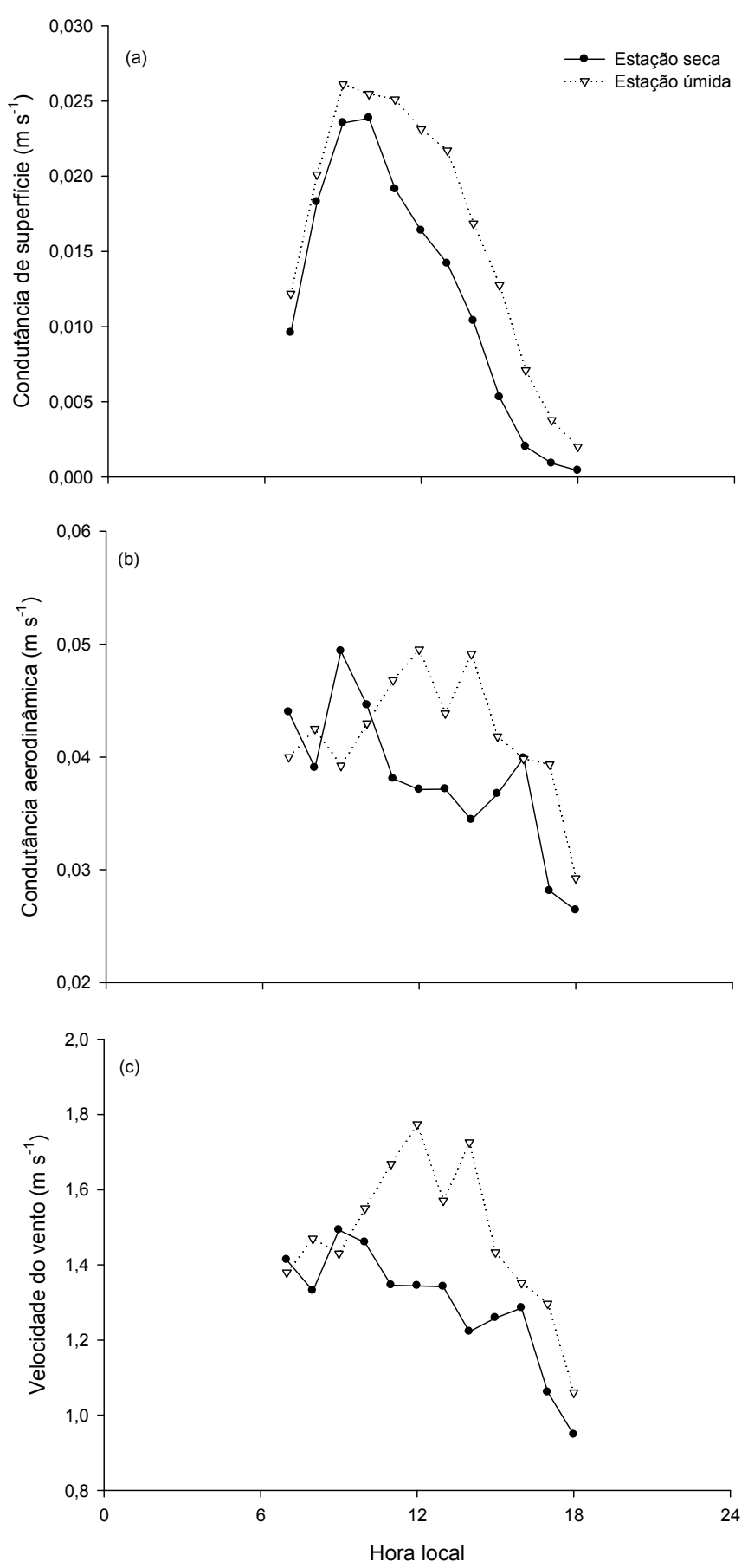

Figura 3: Média mensal $( \pm$ Sd $)$ do (a) déficit de pressão de vapor (DPV), (b) condutância aerodinâmica $\left(\mathrm{C}_{\mathrm{a}}\right)$ e $(\mathrm{c})$ condutância de superfície $\left(\mathrm{C}_{\mathrm{s}}\right)$. 
(1997), quando a magnitude da condutância da camada limite é similar ou menor do que a condutância estomática (no caso deste estudo, que a condutância da superfície), a transpiração das folhas promove a umidificação do ar em torno das folhas, desacoplando a pressão de vapor na superfície da folha da pressão do ar local, situação que possivelmente ocorreu em $V$. divergens na estação úmida. Entretanto, no período seco o $\Omega$ médio foi 0,5 , o que dificultou a compreensão do mecanismo atuante no controle da $\mathrm{ET}_{\mathrm{r}}$.

$\mathrm{O} \Omega$ em $V$. divergens indicou que as condições atmosféricas predominam sobre o controle da evapotranspiração real, e a correlação entre os dados estacionais $(n=5287)$ e o $\Omega$ confirmam que o controle exercido pelo saldo de radiação (Rn) é predominante $\left(\mathrm{R}^{2}=0,43\right.$ na estação úmida e $\mathrm{R}^{2}=0,45$ na estação seca) em relação ao déficit de pressão de vapor (DPV) ( $\mathrm{R}^{2}$ inferior a 0,09 nas duas estações).

\section{CONCLUSÕES}

A evapotranspiração real diminui no período seco em concordância com a diminuição do saldo de radiação. O padrão de variação da condutância de superfície foi semelhante ao verificado em outros estudos em florestas tropicais e em área de floresta alagável.

O fator de desacoplamento foi 0,58 e 0,51 durante as estações úmida e seca, respectivamente, indicando que o controle exercido pelo saldo de radiação sobre a evapotranspiração é predominante. $\mathrm{O}$ desacoplamento entre a cobertura vegetal e a atmosfera durante a estação úmida foi semelhante a estudos em florestas tropicais associado ao índice de área foliar elevado. Estes resultados acrescentam uma dimensão importante para

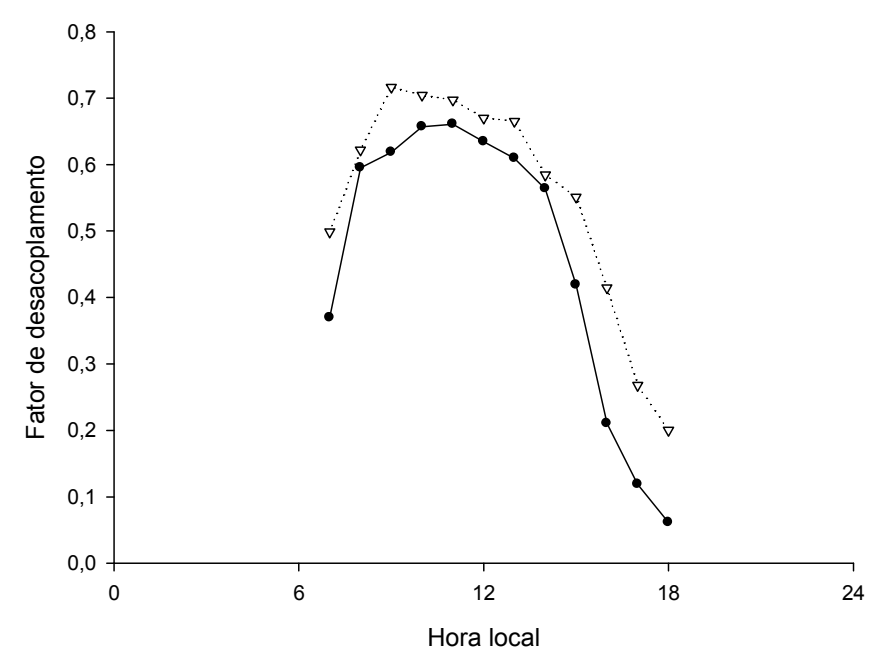

Figura 4: Variabilidade do dia médio estacional do fator de desacoplamento.
Tabela 2: Média mensal $( \pm \mathrm{sd})$ do fator de desacoplamento $(\Omega)^{*}$.

\begin{tabular}{|c|c|c|}
\hline Estação & Mês & $\Omega$ \\
\hline Úmida & Fevereiro & $0,6 \pm 0,2$ \\
\hline Úmida & Março & $0,6 \pm 0,2$ \\
\hline Seca & Abril & $0,7 \pm 0,2$ \\
\hline Seca & Maio & $0,6 \pm 0,2$ \\
\hline Seca & Junho & $0,5 \pm 0,2$ \\
\hline Seca & Julho & $0,5 \pm 0,2$ \\
\hline Seca & Agosto & $0,4 \pm 0,2$ \\
\hline Seca & Setembro & $0,4 \pm 0,2$ \\
\hline Úmida & Outubro & $0,5 \pm 0,2$ \\
\hline Úmida & Novembro & $0,5 \pm 0,2$ \\
\hline Úmida & Dezembro & $0,6 \pm 0,1$ \\
\hline Úmida & Janeiro & $0,6 \pm 0,1$ \\
\hline *Valores considerados entre as 7 e as \\
17 h.
\end{tabular}

a compreensão das características desta floresta inundável reconhecida internacionalmente.

\section{AGRADECIMENTOS}

Este projeto de pesquisa foi desenvolvido com suporte financeiro do Conselho Nacional de Desenvolvimento Científico e Tecnológico (CNPq), Coordenação de Aperfeiçoamento de Pessoal de Nível Superior (CAPES), e suporte adicional da Universidade Federal de Mato Grosso (UFMT).

\section{REFERÊNCIAS BIBLIOGRÁFICAS}

ALVES, I.; PEREIRA, L. S. Modelling surface resistance from climatic variables? Agricultural Water Management, v. 42, p. 371-385, janeiro, 2000.

BURMAN, R. D.; JENSEN, M. E.; ALLEN, R. G. Thermodynamic factors in evapotranspiration. In: Proc., Irrig. and Drain. Spec. Conf. James, L.G; English, M. J. (eds.). Irrigation and Drainage Special Conference ASCE, Portland, Oregon, USA, 1987, p. 28-30.

BUCCI, S. J.; SHOLZ, F. G.; GOLDSTEIN, G.; HOFFMANN, W. A.; MEINZER, F. C.; FRANCO,A.C.; GIAMBELLUCA, T.; MIRALLES-WILHELM, F. Controls on stand transpiration and soil water utilization along a tree density gradient in a Neotropical savanna. Agricultural and Forest Meteorology, v. 148, p. 839-849, junho, 2008.

CAMPBELL, G. S.; J. M. NORMAN. An introduction to environmental biophysics. 2 ed. New York: SpringerVerlag, 1998. $286 \mathrm{p}$. 
CIENCIALA, E.; KUCERA, J.; MALMER, A. Tree sap flow and stand transpiration of two Acacia mangium plantations in Sabah, Borneo. Journal of Hydrology, v. 236, p. 109120, 2000.

COSTA, M. H.; BIAJOLI, M. C.; SANCHES, L.; HUTYRA, L. R.; ROCHA, H. R.; AGUIAR, R. G.; ARAÚJO, A. C. Atmospheric versus vegetation controls of Amazonian tropical rainforest evapotranspiration: Are the equatorial and tropical rainforests any different? Journal of Geophysical Research - Biogeosciences, LBA Special Section, 2007.

DAMASCENO-JUNIOR, G. A.; SEMIR, J.; SANTOS, F. A. M.; LEITÃO-FILHO H.F. Structure, distribution of species and inundation in a riparian forest of Rio Paraguai, Pantanal, Brazil. Flora, v. 200, p. 119-135, maio, 2005.

DAUDET, F. A.; LE ROUX, X.; SINOQUET, H.; ADAM, B. Wind speed and leaf boundary layer conductance variation within tree crown Consequences on leaf-to-atmosphere coupling and tree functions. Agricultural and Forest Meteorology, v. 97, p. 171-185, novembro, 1999.

GRANIER, A.; HUC, R.; BARIGAH, S. T. Transpiration of natural rain forest and its dependence on climatic factors. Agricultural and Forest Meteorology, v. 78, p. 19-29, janeiro, 1996.

GRELLE, A.; LINDROTH, A.; MÖLDER, M. Seasonal variation of boreal forest surface conductance and evaporation. Agricultural and Forest Meteorology, v. 98-99, p. 563-578, dezembro, 1999.

JARVIS, P. G.; MCNAUGHTON, K.G., 1986. Stomatal control of transpiration: scaling up from leaf to region. Advances in Ecological Research, v. 15, p. 1-49, 1986.

KELLIHER, F. M.; LEUNING, R.; RAUPACH, M. R.; SCHULZE, E. D. Maximum conductances for evaporation from global vegetation types. Agricultural and Forest Meteorology, v. 73, p. 1-16, fevereiro, 1995.

KUMAGAI, T.; SAITOH, T. M.; SATO, Y.; MOROOKA, T.; MANFROI, O. J.; KURAJI, K.; SUZUKI, M. Transpiration, canopy conductance and the decoupling coefficient of a lowland mixed dipterocarp forest in Sarawak, Borneo: dry spell effects. Journal of Hydrology, v. 287, p. 237-251, fevereiro, 2004.

LARCHER, W. Ecofisiologia Vegetal. São Carlos: Editora Rima, 2006. 550 p.

LECINA, S.; MARTÍNEZ-COB, A.; PÉREZ, P.J.; VILLALOBOS, F.J.; BASELGA, J.J. Fixed versus variable bulk canopy resistance for reference evapotranspiration estimation using the Penman-Monteith equation under semiarid conditions. Agricultural Water Management, v. 97, p. 181-198, maio, 2003.

MATSUMOTO, K.; OHTA, T.; NAKAI, T.; KUWADA, T.; DAIKOKU, K.; IIDA, S.; YABUKI, H.; KONONOV, A. V.;
VAN DER MOLEN, M.. K.; KODAMA, Y.; MAXIMOV, T. C.; DOLMAN, A. J.; HATTORI, S. Responses of surface conductance to forest environments in the Far East. Agricultural and Forest Meteorology, v. 148, p. 19261940, dezembro, 2008.

McNAUGHTON, K.G.; JARVIS, P.G. Predicting the effects of vegetation changes on transpiration and evaporation. In: Water Deficits and Plant Growth (Kozlowski, T.T.). New York: Academic Press, 1983, v. V, p. 1-48.

MEINZER, F. C.; ANDRADE, J. L.; GOLDSTEIN, G.; HOLBROOK, N. M.; CAVELIER, J.; JACKSON, P. Control of transpiration from the upper canopy of a tropical forest: the role of stomatal, boundary layer and hydraulic architecture components. Plant Cell Environment, v. 20, p. 1242-1252, 1997.

MONTEITH, J.; UNSWORTH, M. Principles of Environmental Physics. Butterworth-Heinemann, 2 ed, 1990, 304 p.

ORTEGA-FARIAS, S. O.; CUENCA, R. H.; EK, M. Daytime variation of sensible heat flux estimated by the bulk aerodynamic method over a grass canopy. Agricultural and Forest Meteorology, v. 81, p. 131-143, setembro, 1996.

PEREIRA, A. R. Introdução à micrometeorologia. Departamento de Física e Meteorologia, ESALQ/USP. Piracicaba, fevereiro, 2000, $63 \mathrm{p}$.

PEREZ, P. J.; CASTELLVI, F.; IBAÑEZ, M.; ROSELL, J. I. Assessment of reliability of Bowen ratio method for partitioning fluxes. Agricultural and Forest Meteorology, v. 97, p. 141-150, novembro, 1999.

PINTO-JÚNIOR, O. B.; SANCHES, L.; LOBO, F. A.; BRANDÃO, A. A.; NOGUEIRA, J. S. Leaf area index of a tropical semi-deciduous forest of the southern Amazon Basin International Journal Biometeorological. doi 10.1007/ s00484-010-0317-1

PRIANTE-FILHO, N.; VOURTILIS, G. L.; HAYASHI, M. M. S.; NOGUEIRA, J. S.; CAMPELO-JUNIOR, J. H.; NUNES, P. C.; SANCHES, L.; COUTO, E. G.; HOEGER, W.; RAITER, F.; TRIENWEILER, J. L.; MIRANDA, E. J.; PRIANTE, P. C.; FRITZEN, C. L.; LACERDA, M.; PEREIRA, L. C.; BIUDES, M. S.; SULI, G. S.; SHIRAIWA, S.; PAULO, S. R.; SILVEIRA, M. Comparison of the mass and energy exchange of a pasture and a mature transitional tropical forest of the southern Amazon Basin during a seasonal transition. Global Change Biology, v. 10, p. 863876, 2004.

RIGHI, E. Z. Balanço de energia e evapotranspiração de cafezal adensado em crescimento sob irrigação localizada. 2004. 151f, Tese (Doutorado em Agronomia), Universidade de São Paulo, 2004.

SANCHES, L., VOURLITIS, G. L., ALVES, M. C., PINTO JR, .O. B., NOGUEIRA, J. S. Seasonal Patterns of 
Evapotranspiration for a Vochysia divergens Forest in the Brazilian Pantanal. Wetlands, v. 31, p. 1215-1225, 2011.

SAN JOSÉ, J. J.; MEIRELLES, M. L.; BRACHO, R.; NIKONOVA, N. A comparative analysis of the flooding and fire effects on the energy exchange in a wetland community (Morichal) of the Oricnoco Llanos. Journal of Hydrology, v. 242, p. 228-254, fevereiro, 2001.

SORIANO, B. M. A; GALDINO, S. Análise da distribuição da freqüência mensal de precipitação para a sub região da Nhecolândia, Pantanal, Mato Grosso do Sul, Brasil. In: EMBRAPA. Centro de Pesquisa Agropecuária do Pantanal (Corumbá, MS). Boletim de Pesquisa e Desenvolvimento 34, 23p, dezembro, 2002.

SOUZA FILHO, J. D. Da C. Variação sazonal dos componentes do balanço de radiação e energia sobre a floresta de Caxiuanã. 2002. 67 f, Dissertação (Mestrado em Meteorologia Agrícola), Universidade Federal de Viçosa, 2002.

STEWART, J. B. Modelling surface conductance of pine forest. Agricultural and Forest Meteorology, v. 43, p. 19-35, junho, 1988.

SHUTTLEWORTH, W. J. Evaporation from Amazonian rainforest. Proceedings of the Royal Society of London, v. 233, p. 321-346, setembro, 1988.

SHUTTLEWORTH, W. J. Terrestrial Hydrometeorology. 1 ed. New York: John Wiley \& Sons, 2012, 448 p.

TAKAGI, K.; TSUBOYA, T.; TAKAHASHI, H. Diurnal hystereses of stomatal and bulk surface conductances in relation to vapor pressure deficit in a cool temperate wetland. Agricultural and Forest Meteorology, v. 91, p. 177-191, junho, 1998.
TODD, R. W.; EVETT, S. R.; HOWELL, T. A. The Bowen ratio-energy balance method for estimating latent heat flux of irrigated alfalfa evaluated in a semi-arid, advective environment. Agricultural and Forest Meteorology, v. 103, p. 335-348, julho, 2000.

UNLAND, E. H.; HOUSER, P. R.; SHUTTLEWORTH, W. J.; YANG, ZONG-L. Surface flux measurement and modeling at a semi-arid Sonoran Desert site. Agricultural and Forest Meteorology, v. 82, p. 119-153, dezembro, 1996.

VOURLITIS, G. L.; NOGUEIRA, J. S. DE; LOBO, F. DE A.; SENDALL, K. M.; DE PAULO, S. R.; DIAS, C. A. A.; PINTO JR O. B; ANDRADE, N. L. R. Energy balance and canopy conductance of a tropical semi-deciduous forest of the southern Amazon Basin. Water Resources Research, v. 44, W03412, doi:10.1029/2006WR005526, 2008

WRIGHT, I.R.; MANZI, A.O.; da ROCHA, H.R. Surface conductance of Amazonian pasture: model application and calibration for canopy climate. Agricultural and Forest Meteorology, v. 75, p. 51-70, junho, 1995.

WULLSCHLEGER, S. D.; MEINZER, F. C.; VERTESSY, R. A. A review of whole-plant water use studies in trees. Tree Physiology, v. 18, p. 499-512, 1998.

WULLSCHLEGER, S. D.; WILSON, K. B.; HANSON, P. J. Environmental control of whole-plant transpiration, canopy conductance and estimates of the decoupling coefficient for large red maple trees. Agricultural and Forest Meteorology, v. 104, p. 157-168, agosto, 2000. 